\title{
Amniotic fluid embolism that took place during an emergent Cesarean section -A case report-
}

\author{
Jung Hyang Lee ${ }^{1}$, Hyeon Jeong Yang ${ }^{1}$, Ji-Hyoung Kim², Su-Yeon Lee ${ }^{1}$, Hyun Jue Gill ${ }^{1}$, Byeong-Kuk Kim ${ }^{1}$ \\ and Min Gu Kim ${ }^{1}$ \\ ${ }^{1}$ Department of Anesthesiology and Pain Medicine, College of Medicine, CHA Univercity, Seongnam, ${ }^{2}$ Goeunmirai Clinic, Incheon, Korea
}

Amniotic fluid embolism (AFE) is a rare but fatal obstetric emergency, characterized by sudden cardiovascular collapse, dyspnea or respiratory arrest and altered mentality, disseminated intravascular coagulation (DIC). It can lead to severe maternal morbidity and mortality, but the prediction of its occurrence and treatment are very difficult. We experienced a case of AFE during emergent Cesarean section in a $40^{+6}$ weeks healthy pregnant woman, age 33. Sudden dyspnea, hypotension, signs of pulmonary edema and DIC were developed during Cesarean section, and cardiac arrest followed after these events. The course of these events was so rapid and catastrophic, which was consistent with AFE. Thus, we report this case precisely and review pathophysiology, diagnosis, treatment of AFE by referring to up-to-date literatures. (Korean J Anesthesiol 2010; 59: S158-S162)

Key Words: Complications; Amniotic fluid embolism, Cardiac arrest, Surgery; Cesarean section.

Amniotic fluid embolism was firstly described in 1926 and set up as a disease entity in 1941 [1,2]. Though rare, it has high maternal morbidity and mortality and is the first cause of maternal death with the varying incidence of $1 / 8,000-1 / 15,200$ [3]. Once it occurs, hypotension and fetal distress take place in all the parturient women, and followed by pulmonary edema, acute respiratory distress syndrome (ARDS), and bleeding due to hemostatic disorder, eventually leading to cardiac arrest [4]. The major factors of the clinical results of amniotic fluid embolism are anaphylaxis, vascular occlusion by particulate matters, vasoconstriction by prostaglandin or others, and disseminated intravascular coagulation [3]. Amniotic fluid embolism usually occurs during parturition at the terminal stage of pregnancy or Cesarean section, but it was reported that amniotic fluid embolism also took place during the dilatation and curettage of missed abortion patients at the second stage of pregnancy [5] and during the cervical suture removal to prevent abortion by incompetent cervix [6]. The diagnosis of amniotic fluid embolism is done clinically and other diseases that should be differentially diagnosed need to be basically excluded. Since there is no lab test available for a definite diagnosis, it is hard to diagnosis amniotic fluid embolism, and once the symptoms

Received: May 10, 2010. Revised: 1st, May 24, 2010; 2nd, June 27, 2010. Accepted: July 2, 2010.

Corresponding author: Hyeon Jeong Yang, M.D., Department of Anesthesiology and Pain Medicine, College of Medicine, CHA Univercity, 351, Yatap-dong, Bundang-gu, Seongnam 463-828, Korea. Tel: 82-31-780-1838, Fax: 82-31-701-9433, E-mail: yanghj@medigate.net

(c) This is an open-access article distributed under the terms of the Creative Commons Attribution Non-Commercial License (http:// creativecommons.org/licenses/by-nc/3.0/), which permits unrestricted non-commercial use, distribution, and reproduction in any medium, provided the original work is properly cited. 
and signs are found, the treatment for amniotic fluid embolism is difficult.

We have experienced amniotic fluid embolism and cardiac arrest that took place during an emergent Cesarean section due to the fetal distress of a healthy parturient woman at the terminal stage of pregnancy. Thus, we report the case herein in reference with the related literature.

\section{Case Report}

The patient was a 33-year-old, $156 \mathrm{~cm}$, and $62.5 \mathrm{~kg}$ primipara on the sixth day of the 40th week of pregnancy, and she was hospitalized on the day before the operation for vaginal delivery. The patient had not undergone any specific disease or operation according to her history, and the vital signs were normal at the time of hospitalization. The results of the blood test and urinalysis performed one month before the hospitalization were all normal. There was no cardiomegaly and abnormal finding in the pulmonary parenchyma by the chest radiography, and the electrocardiography results were all normal. The fetal heart rate measured at the time of hospitalization was $154 \mathrm{bpm}$, and the fetus showed normal reactivity. Although induced labor was carried out by injecting oxytocin during the afternoon on the day of hospitalization, the fetal heart rate was reduced, and thus, the oxytocin injection was stopped. Then, the fetal heart rate returned to normality and natural labor began. When oxytocin was injected again on the day of the operation, the fetal heart rate was reduced. Since meconium staining was found following the amniotic membrane rupture, an emergent Cesarean section was decided. The vital signs that were measured by the electrocardiography, pulse oximetry, and the automated blood pressure device at the operation room were $150 \mathrm{mmHg}$ of systolic blood pressure, $100 \mathrm{mmHg}$ of diastolic blood pressure, 90 of heart rate, and $94 \%$ of oxygen saturation. Because there was no specific finding by the inquiry and auscultation, the low oxygen saturation was considered as an error by the low body temperature since the hands and feet of the parturient woman were cold. Spinal anesthesia was decided because a little dry cough was found. After having the parturient woman take the right lateral decubitus position, $0.5 \%$ hyperbaric bupivacaine $10 \mathrm{mg}$ mixed with fentanyl 20 ug was injected with a $26 \mathrm{G}$ spinal needle between the L3 and L4 spines. The cold sense check was performed with an alcohol swab to verify the sensory block level to the fourth thoracic spinal segment. Five minutes after the spinal anesthesia, blood pressure was decreased to 90/50 $\mathrm{mmHg}$, and the pulse oxygen saturation to $88 \%$. The parturient woman complained of severe dyspnea and a continued cough and a large amount of sputum mixed with pink bubbles were observed. After ephedrine was immediately injected 3 times, 8 $\mathrm{mg}$ each, the blood pressure was recovered to $110 / 80 \mathrm{mmHg}$, the heart rate to $90 / \mathrm{min}$, and the oxygen saturation to $92 \%$, and then the operation was initiated. The results of the arterial blood gas study at that moment were $\mathrm{pH}$ 7.39, oxygen partial pressure $\left(\mathrm{PaO}_{2}\right) 77.7 \mathrm{mmHg}$, carbon dioxide partial pressure $\left(\mathrm{PaCO}_{2}\right) 34.5 \mathrm{mmHg}$, and oxygen saturation 95\%. Just after the start of the operation, the spontaneous breathing seemed no longer impossible due to the severe dyspnea and excessive airway secretion. Thus, after injecting propofol $60 \mathrm{mg}$ and succunycholine $70 \mathrm{mg}$, endotracheal intubation was carried out with the endotracheal tube of the inner diameter $7.0 \mathrm{~mm}$. After the endotracheal intubation, a large amount of pink discharge was continuously aspirated through the tube to the extent that it blocked the tube, and the oxygen saturation of the patient was decreased down to $70 \%$. Following the laparotomy, a great quantity of ascites was observed. After the fetus was delivered, the blood pressure was decreased to $60 / 40 \mathrm{mmHg}$, the oxygen saturation to $65 \%$, and the heart rate to $60 / \mathrm{min}$. Although dobutamine and norepinephrine were injected since heart failure was suspected, pulseless electrical activity (PEA) was found and the operation was suspended temporarily to carry out cardiopulmonary resuscitation. After the injection of epinephrine $1 \mathrm{mg}$ and atropine $0.5 \mathrm{mg}$ with continued thoracic compression, the sinus rhythm was recovered and the vital signs were increased to the blood pressure of 150/80 $\mathrm{mmHg}$, oxygen saturation of $95 \%$, and heart rate of $120 / \mathrm{min}$. With the continuous intravenous injection of norepinephrine, as well as dopamine and dobutamine, a central vein catheter was inserted through the right internal jugular vein and an arterial catheter through the left femoral artery. Although the operation was then resumed, a severe hemorrhage was found and the blood pressure was reduced to $60 / 40 \mathrm{mmHg}$ and the oxygen saturation to $80 \%$ at the end of the operation. The results of the arterial blood gas study at that moment were pH 7.154, $\mathrm{PaO}_{2} 63.5 \mathrm{mmHg}, \mathrm{PaCO}_{2} 57.5 \mathrm{mmHg}$, bicarbonate $19.8 \mathrm{mmol} / \mathrm{L}$, and oxygen saturation $85.1 \%$, which indicated a serious respiratory and metabolic acidosis and hypoxia. An electrolyte disorder was also observed as the ionized calcium concentration was $0.6 \mathrm{mmol} / \mathrm{L}$ and that of the potassium was $5.78 \mathrm{mmol} / \mathrm{L}$. Bicarbonate was injected to correct the acidosis and the $50 \%$ dextrose in water $(\mathrm{D} / \mathrm{W})$ solution to which $\mathrm{CaCl}_{2}$ $600 \mathrm{mg}$ and insulin were added was also injected. In addition, pentaspan and normal saline were continuously injected through the central vein. Since the possibility of hemorrhage by coagulation disorder, not the surgical hemorrhage, could not be excluded, the packed red blood cells and the fresh frozen plasma were transfused, too. Although the blood test related with disseminated intravascular coagulation (DIC) was carried out during the operation (bleeding time, prothrombin time, activated prothromin time, antithrombin III, fibrinogen, D-dimer, and fibrinogen degradation product), the results 
could not be obtained because of coagulation of the blood sample. Even after the finish of the operation, the low blood pressure was maintained and the patient returned to the PEA state. Cardiopulmonary resuscitation was performed and epinephrine $1 \mathrm{mg}$ and atropine $0.5 \mathrm{mg}$ were injected 2 times for each. After the patient was recovered to the sinus rhythm, she was moved to the intensive care unit. The total operation duration was 1 hour; the urination was $150 \mathrm{ml}$; the blood loss was $1,500 \mathrm{ml}$; the injected fluid was $1,850 \mathrm{ml}$; and the transfused blood was 1,250 $\mathrm{ml}$. More than $3,000 \mathrm{ml}$ of secretion was discharged through the endotracheal tube and it spouted out continuously even in the intensive care unit. Heart failure took place once again and cardiopulmonary resuscitation was performed continuously. However, the blood pressure was dropped to $53 / 30-40 / 20 \mathrm{mmHg}$, the heart rate to $20-30 / \mathrm{min}$, and the oxygen saturation to $50-60 \%$. The complete heart failure was continued as the patient's status did not become any better, and the patient showed no response to the medications. Though the cardiac compression was continually carried out, the patient died after 3 hours. The autopsy was performed by the National Institute of Scientific Investigation and the cause of death was proved to be amniotic fluid embolism.

\section{Discussion}

Amniotic fluid embolism is a fatal syndrome that takes place during pregnancy or during or after delivery. Amniotic fluid embolism was firstly described in 1926 [1], and it was recognized as a syndrome by Steiner and Lushburgh [2] as fetal debris were found within the pulmonary blood vessels of the parturient women who died during labor by similar clinical characteristics. Although it was reported that the incident rate was 1 out of $8,000-15,200$ live births [3] and the mortality was in the range of $61-86 \%[3,4]$, the mortality was decreased to $13.3-44.0 \%$ according to a recent study. It is thought that this trend may be because the diagnosis and treatment have been advanced and only fatal cases were reported selectively. Amniotic fluid embolism holds 5-15\% of the overall causes of maternity deaths, and it leaves permanent neurological damage to $61 \%$ of the parturient women and $50 \%$ of the newborn children [4]. Many have been known as the risk factors of amniotic fluid embolism, but parturient women over the age of 35, Cesarean section, forceps and fetal suction, placenta previa, abruptio placentae, eclampsia, and fetal distress syndrome were verified as the risk factors by a large scale research that was carried out recently although the correlation among them has not been understood clearly [7]. The major clinical characteristics include cardiovascular collapse accompanied by severe hypotension and arrhythmia, cyanosis, respiratory distress, pulmonary edema or acute respiratory failure syndrome, respiratory arrest, consciousness fluctuation, and massive hemorrhage by DIC. Among these, the incidents of hypotension, respiratory failure, and cyanosis is almost $100 \%$. The major causes of maternal death are cardiac arrest, massive hemorrhage by DIC, acute respiratory failure syndrome, and multiple organs dysfunction. According to the report by Lewis [8], 11 of the 17 parturient women who had experienced amniotic fluid embolism complained of prodromal symptoms such as dyspnea, chest pain, chilliness, restlessness, the feeling of being pierced by a pin, nausea and vomiting. The time interval between the observations of these symptoms to the women's collapse varied, ranging from several minutes to 4 hours. In this case report, even though the parturient woman showed the symptom of dry coughing from the morning of the operation day onwards, it was not very severe and no other specific symptom was observed. The causes of the prophase hypoxia, which is the most representative clinical characteristic of amniotic fluid embolism, are severe ventilation-perfusion mismatch by pulmonary vasoconstriction and bronchospasm, and those of the anaphase hypoxia include pulmonary edema by left ventricular failure and nonpsychogenic pulmonary edema that is related to the increase of the capillary permeability. Suchlike hypoxia may be the cause of heart failure and neurologic damage. The parturient woman in this case report showed an oxygen saturation of $94 \%$ at the time of entering the operation room, which might have been the indication of hypoxia that occurred already, rather than the error by hypothermia. In addition, from the chest radiograph taken in the operation room after the fetus was delivered (Fig. 1), severe pulmonary edema at both lungs and normal central venous pressure were found, though the pulmonary arterial

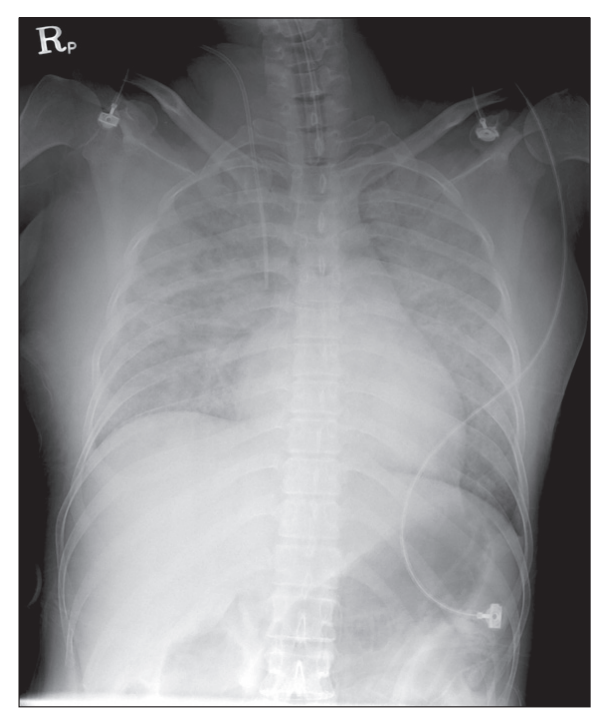

Fig. 1. Chest X ray, anterior-posterior view after baby out during cesarean section. 
pressure was not measured. Thus, it is assumed that the hypoxia might have been caused by pulmonary edema due to the left ventricular failure or the increase of the pulmonary capillary permeability, rather than by the pulmonary vasoconstriction at the initial stage. The acute cardiovascular collapse can be explained by the biphasic model suggested by Clark [9]. Right ventricular failure can take place at the initial stage by the pulmonary vasoconstriction and pulmonary hypertension due to the secretion of endogenous mediators, which was proved in a number of reports. Shechtman et al. [10] reported that acute right ventricular failure accompanied by severe pulmonary hypertension, left deviation of the atrial, and ventricular septum was found from the transesophageal echocardiography performed within 30 minutes after the occurrence of amniotic fluid embolism. A large amount of ascites was also found after the laparotomy in our case, which is thought to be the secondary result of the right ventricular failure. As the operation moved on to the anaphase, the pulmonary hypertension did not continue, but it was shifted to left ventricular failure, which might have been caused mainly by the decreased filling of the left ventricle following the enlargement of the right ventricular enlargement. Other causes of myocardial failure that are known include myocardial ischemia due to hypoxia, decreased blood flow in coronary artery due to decreased cardiac output, and direct myocardial depression by the substances in amniotic fluid such as endothelin. The blood pressure of the parturient woman in our report was decreased after the spinal anesthesia, which could have resulted from the high level of sensory block to the T4 spine, but it could be probably because of the decrease of the general vascular resistance following the left ventricular failure. DIC is also one of the factors that can cause hypovolemic shock, and it is found in $50 \%$ of the parturient women with amniotic fluid embolism. The mechanism of DIC is not clear, but it is known by a previous animal experiment that amniotic fluid is related to the thromboplastin-like effect, platelet aggregation, and the activation of complement reaction. In addition, Lockwood et al. [11] discovered a large amount of tissue factors in amniotic fluid and explained the triggering of blood coagulation and consumptive coagulopathy caused by the activation of extrinsic pathway and factor X. DIC, which is clinically characterized by continuous blood loss, can take place in any stage of amniotic fluid embolism, from the initial stage to the terminal stage. In this case report, the heart was temporarily recovered by the cardiopulmonary resuscitation after the cardiac arrest, but the prognosis could become worse as hypovolemic shock took place due to the DIC afterward. In conclusion, the main causes of the maternal death of the parturient woman are thought to be the hypotension by the combined effect of the left ventricular failure, the decrease of the general vascular resistance due to the spinal anesthesia and continued blood loss by DIC, and pulmonary failure by the severe pulmonary edema. The etiology of amniotic fluid embolism is not clear yet. The pathway of amniotic fluid influx to the maternal circulatory system includes the uterine cervical vein, damaged uterine site, and placental site. In early studies, pulmonary vascular occlusion was considered as the main etiology of amniotic fluid embolism, but various clinical characteristics of amniotic fluid embolism were hardly explained by the mechanism and it was not verified by animal experiment [12], implying that there might be another mechanism rather than the mechanical occlusion. Hammerschmidt et al. [13] mentioned that activation of complements and granulocytes caused the pulmonary vascular occlusion. Now, the secretion of primary or secondary endogenous mediators following the amniotic fluid inflow to the maternal circulatory system is considered as the major etiology of amniotic fluid embolism based on several reports. The known mediators include histamine, bradykinin, endothelin, leukotriene, and arachidonic acid metabolites. These immunological factors can be supported by the fact that amniotic fluid embolism is found more frequently among the parturient women who have conceived a male fetus and with the history of drug allergy.

Amniotic fluid embolism is diagnosed clinically after excluding other diagnoses. In other words, amniotic fluid embolism can be strongly suspected in a pregnant woman or a puerperal woman, immediately after the delivery, with cardiovascular collapse as well as respiratory failure, DIC, and convulsion, excluding anaphylaxis, septisemia, pulmonary embolism, myocardial infarction, perinatal cardiomyopathy, and hemorrhagic shock (atony, uterine rupture, and abruptio placentae). Blood test, chest radiography and echocardiography can help, but they are nonspecific. Although in many studies amniotic fluid tissue was found in the blood aspirated at the terminal of the pulmonary artery catheter installed in parturient women who were diagnosed as having amniotic fluid embolism [14], the amniotic fluid tissue was found in only about $50 \%$ of the parturient women in the study of Clark et al. [4], as well as in other studies. Moreover, the component of amniotic fluid is also found among the women who do not have amniotic fluid embolism or the women who are not pregnant. Hence, that a component of amniotic fluid is found in a maternal circulatory system does not necessarily mean that amniotic fluid embolism has occurred. However, the probability of amniotic fluid embolism is increased in the case where there are the clinical characteristics that suggest amniotic fluid embolism. Also, in the parturient woman in this case, the diagnosability was increased since a great amount of amniotic fluid component was found in the pulmonary blood vessels, as well as the clinical characteristics of amniotic fluid 
embolism. Beside this, another known method of amniotic fluid embolism diagnosis is to measure zinc coproporphrin, sialyl Tn antigen, tryptase, and complement factors in the peripheral blood of a parturient woman, but more research is required for it. The treatment is performed symptomatically depending on the symptoms of the patient and the basic direction of the treatment is maintenance of the appropriate oxygenation level and blood pressure, and correction of the coagulopathy. Firstly, for the hypoxia, tracheal intubation should be immediately carried out and positive-pressure ventilation with oxygen of high concentration should be performed so as to maintain the oxygen saturation higher than $90 \%$. For the hypotension and the shock, the preload should be increased by means of rapid injection of a crystalloid solution. Since an overdose of fluid can worsen the heart failure at this time, it is helpful to monitor by means of pulmonary catheter or electrocardiography. In the case of a serious hypotension which does not respond to a fluid treatment, vasopressors such as norepinephrine or cardiac inotropic agents such as dopamine, dobutamine, and milrinone can be used. When a large amount of hemorrhage occurs due to DIC, packed red blood cells should be primarily transfused to supply oxygen to the tissue appropriately. Platelets, fresh frozen plasma, and cryoprecipitate also need to be transfused. Among them, cryoprecipitate has been known to help the patient to recover the cardiopulmonary and hematological state rapidly by enhancing the removal of antigenic and toxic substances such as amniotic fluid since it contains fibronectin. When cardiopulmonary arrest takes places during the clinical course, cardiopulmonary resuscitation should be immediately carried out and cesarean section should be performed as early as possible in order to improve the prognosis of the parturient woman and the fetus. Since the gravid uterus represses the venous return by the aortocaval compression, quick delivery of the fetus makes the cardiopulmonary resuscitation more effective. In addition to these, other treatments are available including aprotinin, serine proteinase inhibitor, cardiopulmonary bypass, pulmonary embolectomy, hemofiltration, and inhalation of nitric oxide gas, etc. Recently, a case where extracorporeal membrane oxygenation showed a good result in a parturient woman with amniotic fluid embolism who had a cardiac arrest was reported, and it was newly suggested as a treatment [15].

In conclusion, as experienced in this case, amniotic fluid embolism is rare, but it is a rapidly developing, fatal disease. However, this disease is hard to diagnose in its onset, and the treatment is still difficult. Thus, a prompt and positive treatment should be carried out if there is a parturient woman in whom a sudden cardiopulmonary collapse, respiratory failure, and hemorrhage are found. Particularly when cardiac arrest takes place, cardiopulmonary resuscitation should be carried out immediately and the fetus should be delivered at the same time in order to improve the prognosis of the parturient woman and the fetus. It must be noted that even after the parturient woman has recovered from the cardiac arrest, neurologic damage caused by hypoxia can still take place.

\section{References}

1. Meyer J. Embolia pulmonar amnio caseosa. Bras Med 1926; 2: 301.

2. Steiner P, Luschbaugh C. Maternal pulmonary emboli by amniotic fluid. JAMA 1941; 117: 1245-54.

3. Morgan M. Amniotic fluid embolism. Anaesthesia 1979; 34: 20-32.

4. Clark SL, Hankins GD, Dudley DA, Dildy GA, Porter TF. Amniotic fluid embolism: analysis of the national registry. Am J Obstet Gynecol 1995; 172: 1158-69.

5. Kim BI, Paek SH, Rho WS, Lee SP, Cho SK, Lee SH. Amniotic fluid embolism during dilatation and curettage in a second trimesteric missed aborted pregnant patient. Korean J Anesthesiol 1997; 33: 778-83.

6. Haines J, Wilkes RG. Non-fatal amniotic fluid embolism after cervical suture removal. Br J Anaesth 2003; 90: 244-7.

7. Abenhaim HA, Azoulay L, Kramer MS, Leduc L. Incidence and risk factors of amniotic fluid embolisms: a population-based study on 3 million births in the United States. Am J Obstet Gynecol 2008; 199: 49.e1-8.

8. Lewis G. The confidential enquiry into maternal and child health (CEMACH). Saving mothers' lives: reviewing maternal deaths to make motherhood safer--2002-2005. The seventh report on confidential enquiries into maternal deaths in the United Kingdom. London: CEMACH; 2007.

9. Clark SL. New concepts of amniotic fluid embolism: a review. Obstet Gynecol Surv 1990; 45: 360-8.

10. Shechtman M, Ziser A, Markovits R, Rozenberg B. Amniotic fluid embolism: early findings of transesophageal echocardiography. Anesth Analg 1999; 89: 1456-8.

11. Lockwood CJ, Bach R, Guha A, Zhou XD, Miller WA, Nemerson Y. Amniotic fluid contains tissue factor, a potent initiator of coagulation. Am J Obstet Gynecol 1991; 165: 1335-41.

12. Stolte L, van Kessel H, Seelen J, Eskes T, Wagatsuma T. Failure to produce the syndrome of amniotic fluid embolism by infusion of amniotic fluid and meconium into monkeys. Am J Obstet Gynecol 1967; 98: 694-7.

13. Hammerschmidt DE, Ogburn PL, Williams JE. Amniotic fluid activates complement. A role in amniotic fluid embolism syndrome? J Lab Clin Med 1984; 104: 901-7.

14. Resnik R, Swartz WH, Plumer MH, Benirschke K, Stratthaus ME. Amniotic fluid embolism with survival. Obstet Gynecol 1976; 47: 295-8.

15. Ho CH, Chen KB, Liu SK, Liu YF, Cheng HC, Wu RC. Early application of extracorporeal membrane oxygenation in a patient with amniotic fluid embolism. Acta Anaesthesiol Taiwan 2009; 47: 99102. 